\title{
The Prognostic Ability of Staging System in Men with Penile Cancer: An Analysis of SEER Database
}

\author{
Aditya Jalan', Ravi Kanodia ${ }^{2}$, Sarita Rana Gurung1, Rajeev Kumar Malhotra ${ }^{3}$, Umesh Nepal', Gyan \\ Prasad Pokhrel ${ }^{1}$, Nirmal Lamichhane ${ }^{1}$ \\ ${ }^{1}$ Department of Surgical Oncology, B.P. Koirala Memorial Cancer Hospital, Bharatpur, Chitwan, Nepal. \\ ${ }^{2}$ Department of Radiation Oncology, All India Institute of Medical Sciences, New Delhi, India. \\ ${ }^{3}$ Department of Delhi Cancer Registry, All India Institute of Medical Sciences, New Delhi, India.
}

\begin{abstract}
Background: Penile cancer is now a rare condition. The low incidence of the disease makes a valid estimation of its prognosis difficult. In this study, we made an attempt and propose a nomogram to develop a prognostic rule that could predict the Cancer-Specific Mortality (CSM) free rates in patients with primary penile squamous cell carcinoma of the penis (PPSCC).

Methods: This study included 1304 patients diagnosed with PPSCC between the years $2004 \& 2011$ and treated with penile tumor excision. Subjects were staged as per Surveillance, Epidemiology \& End Results stage (SEER), American Joint Committee on Cancer (AJCC), TNM classification and tumor grade (TG). CSM free rates were determined. Univariate and multivariate Cox regression model was used to test the prediction of the CSM free rate. The predictive rule accuracy was created using the receiver operating characteristic curve.
\end{abstract}

Results: The clinico-pathological profile depicts a mean age of $64.66 \pm 14.38 \mathrm{yrs}$. The most common primary site involved was glans penis $(n=483,37 \%)$ and the disease was most commonly diagnosed at AJCC stage I $(n=670$, 51.4\%) disease. The cumulative 5-year CSM free rates according to Fine \& Gray, \& Kaplan-Meier methods were $81.8 \%$ and $79.8 \%$, respectively. The predictive accuracy as per SEER stage, AJCC stage, TNM stage alone were $68.8 \%, 70.3 \%, 72.3 \%$, respectively. When TG was combined, the predictive accuracy increased to $72.8 \%, 73.1 \%$, and $75.0 \%$, respectively. TNM stage with TG was most accurate in predicting CSM free rate compared to other models.

Conclusions: TNM stage with TG and AJCC stage with TG appear to have comparable accuracy to predict the CSM free rate in patients with PPSCC, the TNM stage with TG is the most accurate (75\%) method to predict the CSM free rates. The addition of the TG variable improved the accuracy of these prognostic models.

Keywords: Normograms, Penis, Squamous Cell Carcinoma, Survival

\section{Introduction}

Penile cancer is now a rare condition, accounting for less than $1 \%$ of cancers diagnosed in men in the United States. Most of the penile cancers are squamous cell carcinoma. Though rare, the disease itself is a challenge for urologist and onco-surgeons as it carries significant morbidity and mortality with approximately 50\%, 5-year cancer-specific survival. ${ }^{1}$ With an improvement in the understanding of the natural history of the disease, the cure rate has increased from $50 \%$ in 1990 to $80 \%$ in recent

Correspondence

Aditya Jalan, Address: Department of Surgical Oncology, B.P. Koirala Memorial Cancer Hospital, Bharatpur, Chitwan, Nepal.Email: dradityajalan@gmail.com 
years. ${ }^{2}$ Lower incidence of the disease makes the valid estimation of prognosis of primary penile squamous cell carcinoma (PPSCC) difficult. To address this issue many investigators Kattan et al, Zini et al, and Thuret et al developed various models to predict Cancer-Specific Mortality (CSM) free rates for PPSCC..$^{3-5}$

The pathological presence of lymph node metastases is the most powerful predictor of cancer-specific survival in patients with PPSCC. ${ }^{6}$ In clinically lymph nodenegative patients, this information is only available in those patients when they undergo inguinal lymph node dissection (ILND). However, due to a higher incidence of postoperative local complications all the patients do not undergo ILND. Evidence suggests though micrometastasis may be present in up to $25 \%$ of patient with clinically negative nodes. ${ }^{7}$

In this present study, we made an attempt to reconfirm the findings of the previous studies after many years now and propose a nomogram to develop a prognostic rule that could be applied to US men to predict the CSM free rates in patients with PPSCC after penile tumor excision (PTE).

\section{Methods}

The Surveillance, epidemiology and end results (SEER) database consisting of 18 SEER registries of the National Cancer Institute (NCI) program covers approximately $34.6 \%$ of the US population and is considered the representative of the United States with regard to demographic composition, as well as of cancer incidence and mortality. In this study, it was used to extract information on subjects diagnosed with PPSCC between the years 2004 and 2011. Patients were identified according to diagnostic codes: the tenth revision of the International Classification of Disease (ICD) for Oncology [C60.0-60.9] and the ICD-O-3 codes for histological subtype (squamous cell carcinoma type; ICD-O-3: 8050-8089). All subjects were staged according to the American Joint Committee on Cancer (AJCC) sixth edition (2002), 2002 TNM system based on the SEER Extent of Disease Classification. Variables analyzed were: Age, race, marital status, tumor stage and grade, TNM stage distribution and SEER registry. The extent was grouped as localized (cancer was limited to the penis), regional (cancer extended outside the penis to lymph nodes in the pelvic area), distant metastasis.

The point of observation was from PTE. Only patients who had PTE were included in the study. Those who didn't undergo surgery or when the surgical procedure was not specified or when surgery type was unknown were excluded. Patients with unknown SEER Stage, AJCC stage, unknown grade, and unknown metastatic status were also excluded. Similarly, the number of patients from Alaska Natives and Rural Georgia were very less and were also excluded ( detailed in Figure 1). Patients whose nodal status was not accessed were considered as N0. The patients in whom T stage and M stage could not be accessed were also less in number, and were excluded. The cause of death was defined according to SEER specific cause of death codes. And for the analysis, death from other cause was considered as censored or as other cause mortality on competing risk analysis. (Figure 1)

Descriptive statistics were used to summarize the cohort, including mean $\pm \mathrm{SD}$, median and range for continuous variables, or counts and percentages for categorical variables. Kaplan-Meier plots were performed to determine CSM-free survival rates in the study population, and after stratifying by SEER and AJCC stage, TNM classification and TG. Log-rank test was performed to compare the categories within AJCC stage, TNM, and TG and p-value was adjusted as per Bonferroni corrections.

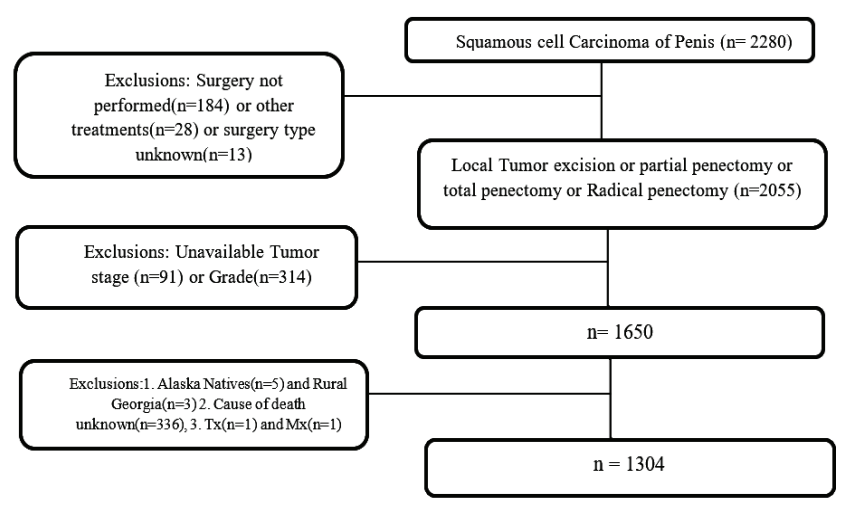

\section{Figure 1: Flow chart describing inclusion and exclusion criteria of patients}

Three multivariable proportional hazard Cox regression models were used to test the accuracy of SEER stage vs AJCC stage vs TNM classification to predict CSM. The 
proportionality assumption was tested using Schoenfeld residual graphs and also by the statistical test. An additional ${ }^{3}$ Cox regression models were fitted using the same disease stages after adding of TG. Likelihood ratio test was applied to test the additional advantage of TG. These models were used to develop nomograms. The prognostic ability of nomogram was quantified with receiver operating characteristic (ROC) curve derived area under the curve (AUC) estimates. The prognostic ability of the 3 staging systems with and without TG was tested for predicting the 5-year CSM-free rate.

Since a proportion of patients with PPSCC die of other causes, we used univariate and multivariate competing risks regression models, as described by Fine and Gray, to test the significance of the variables in predicting CSM free rates. ${ }^{8}$

Competing risks regression models allow us to account for the effect of other cause mortality. All statistical analyses were performed using SEER ${ }^{\star}$ Stat (version 8.3.5; National Cancer Institute, Bethesda,Md) and survival curves were generated with the survival function from the $\mathrm{R}$ statistical package for Windows version 3.5.2. All statistical tests were done with $\mathrm{R}$ statistical package. Statistical significance was set at 0.05 .

\section{Results}

The clinicopathological profile of all patients of PPSCC who underwent PTE between 2004-2011 is summarized in Table 1 . The mean age was $64.66 \pm 14.38$ years and most were married ( $n=762,58.4 \%)$. Most of the men belong to the white race $(n=1094,83.9 \%)$. The most common primary site involved was glans penis $(n=483$, 37\%). According to SEER stage, the disease was localized in most of the patients $(n=777,59.6 \%)$. Majority $(n=719$, $55.1 \%$ ) of cases underwent partial penectomy followed by local tumor excision in $(n=372,28.5 \%)$ of patients. Most were confirmed as moderately differentiated grade II followed by well-differentiated grade squamous cell carcinoma. However, lympho-vascular invasion was identified only in $4.8 \%(n=63)$ of patients. Most common pathological T stage was T1 $(56.7 \%, \mathrm{n}=740)$, $\mathrm{N}$ stage was N0 $(\mathrm{n}=1055,80.9 \%)$ and M stage was M0 $(\mathrm{n}=1269,97.3 \%)$. Most commonly diagnosed AJCC was stage I $(n=670,51.4 \%)$.
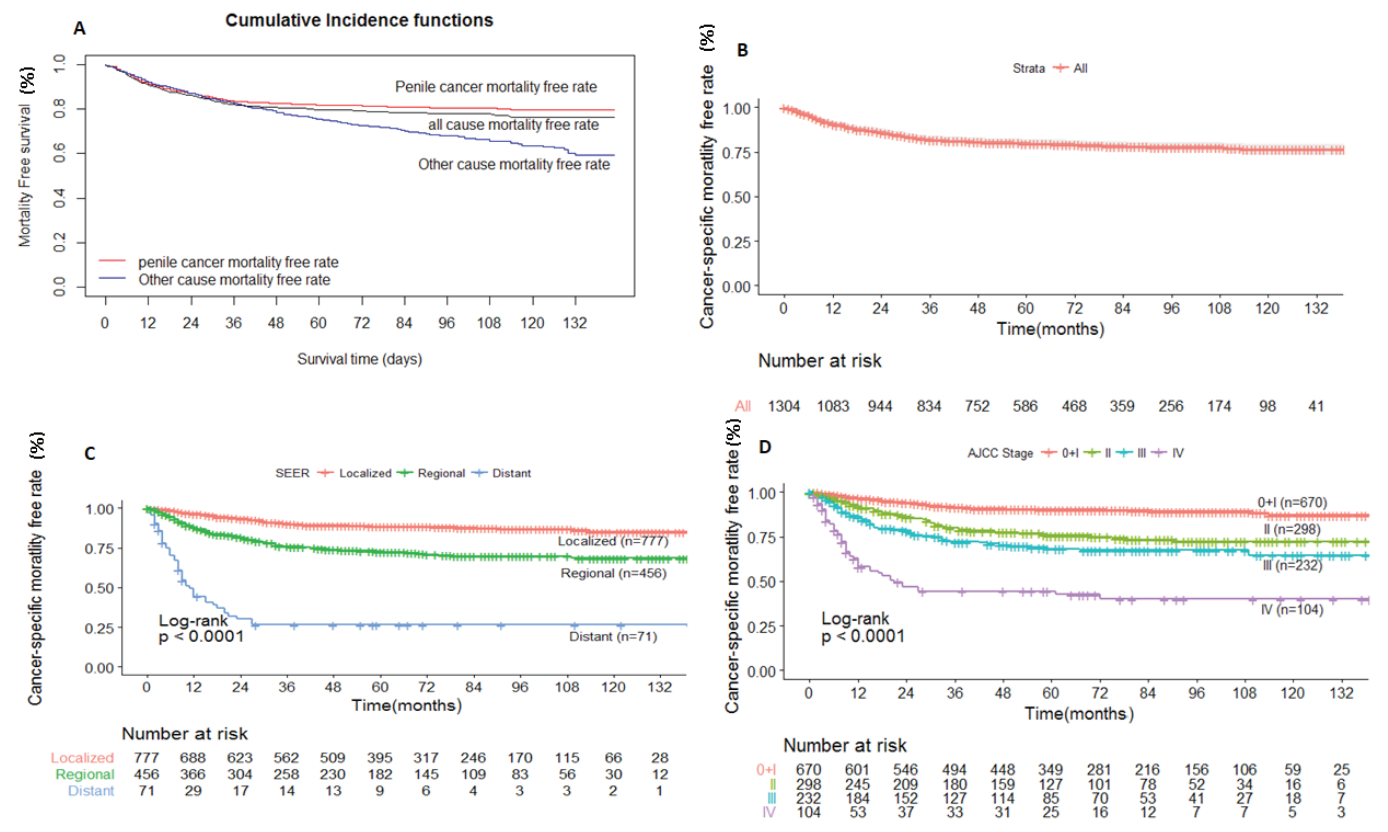

Figure 2: Cumulative incidence graphs shows CSM-free and other cause mortality-free rates in overall population of 1,304 men (A). Kaplan-Meier survival curve represents CSM-free rate in overall cohort (B). Kaplan-Meier survival curve shows CSM-free rate by localized vs regional vs distant SEER stage (C). Kaplan-Meier survival curve demonstrates CSM-free rate AJCC stages I vs II vs III vs IV (D). 
Figure 2 ( $A$ and $B$ ) shows the cumulative 5-year CSM free survival rates according to Fine and Gray, and according to Kaplan-Meier method. Both the graphs were virtually superimposed. The Cumulative 5-year CSM incidencefree rates according to Fine and Gray, and according to Kaplan-Meier method was $81.8 \%$ and $79.8 \%$ respectively. Figure $2(C$ and $D)$ and figure $3(A-D)$ shows 5 years CSM free rate according to different groups. In the SEER stage localized, regional and distant CSM free rates were found to be $88.3 \%, 72.7 \%$, and $26.6 \%$ respectively. According to AJCC 6th ed, stage I/0, stage II, stage III and stage IV were $90.3 \%, 75.7 \%, 68.3 \%, 42.6 \%$ respectively. After stratifying TNM staging, T1/Ta, T2, T3 and T4, 5 years CSM free rates were $86.7 \%, 72.0 \%, 66.5 \%$ and $80.0 \%$ respectively. Likewise, CSM free rate for nodal stage N0, $\mathrm{N} 1, \mathrm{~N} 2$ and $\mathrm{N} 3$ were $86.6 \%, 55.8 \%, 47.6 \%$ and $35.4 \%$ respectively. Those cases with no metastasis had CSM free rate of $81.2 \%$. According to tumor grading, CSM free rate in grade I, II and III were $90.4 \%, 77.7 \%$, and $66.6 \%$ respectively. All log ranks were statistically significant except T1a vs T4, T2 vs T3, T2 vs T4, T3 vs T4 and N1 vs $\mathrm{N} 2$ after Bonferroni corrections.

The Cox regression analysis was done to generate a table predicting individualized CSM probabilities at 5-year after surgical removal of PPSCC (Table 2). Most of the variables were found to be statistically significant (except T1 vs T4). The predictive accuracy as per SEER stage, AJCC stage, TNM stage were $68.8 \%, 70.3 \%, 72.3 \%$ respectively. Similarly, the predictive accuracy of TG alone was $64.0 \%$. However, when the SEER stage, AJCC stage, and TNM stage were combined with TG there was an increase in predictive accuracy which resulted in $72.8 \%, 73.1 \%$, and $75.0 \%$ respectively. When the SEER stage, AJCC Stage and TNM stage were analyzed in a combination of TG stage and found the contribution of TG in the presence of above factor was found to be statistically significant. There was an increment of $4 \%$ in SEER stage, 2.8\% in AJCC stage and 2.7\% in TNM staging in AUC when TG was added in the model.

The competing risk regression model was used to adjust the patients for non-penile cancer-related mortality (Table 3). The competing risk model for predictors of CSM free mortality SEER stage, AJCC stage, TNM stage, and TG stage was observed to be statistically significant. When each stage was combined with TG stage it was still found to be significant except for T4 versus T1.
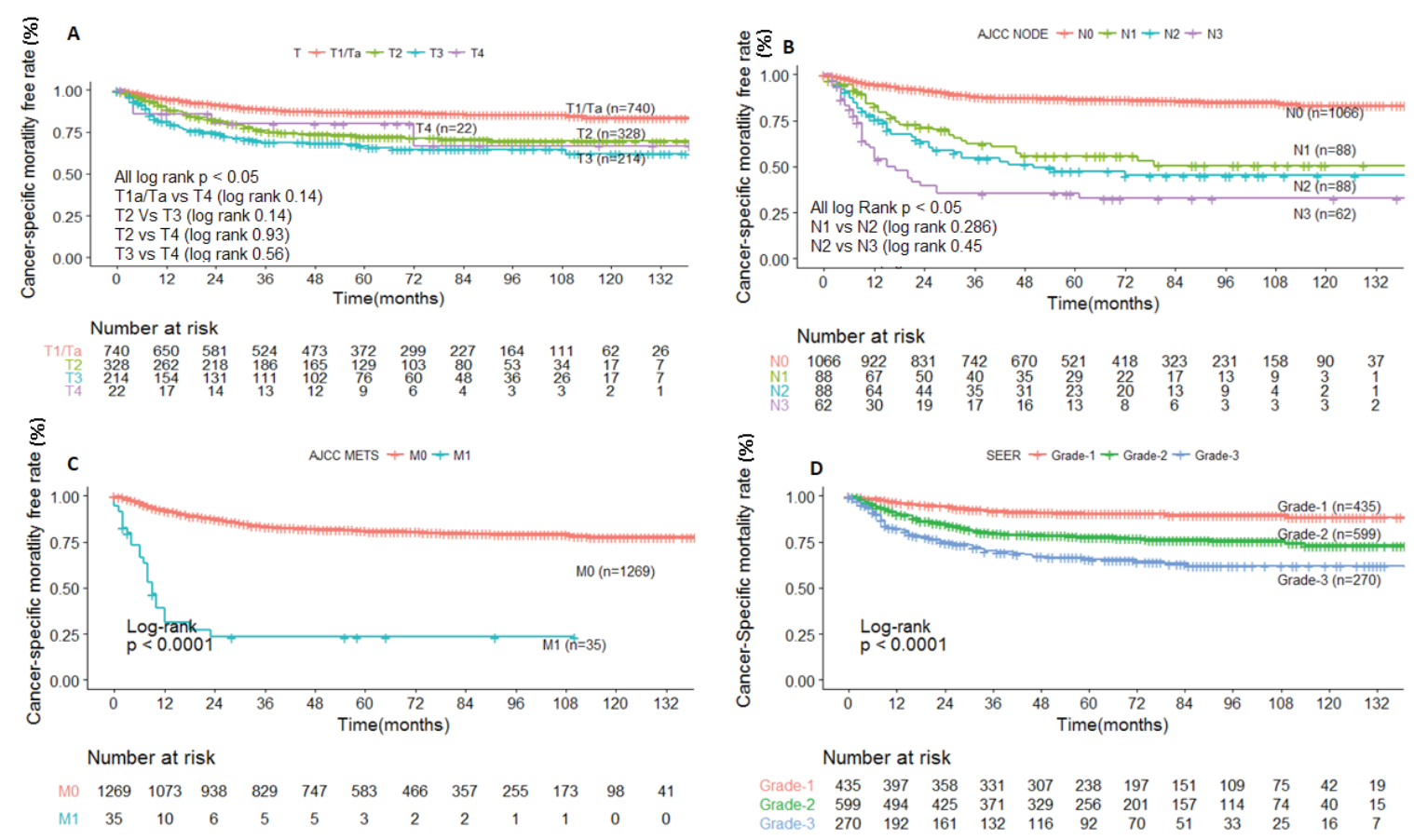

Figure 3: Kaplan-Meier plots show CSM-free rate after stratification by $T$ (A), N (B) and M (C) stages, and TG (D). 

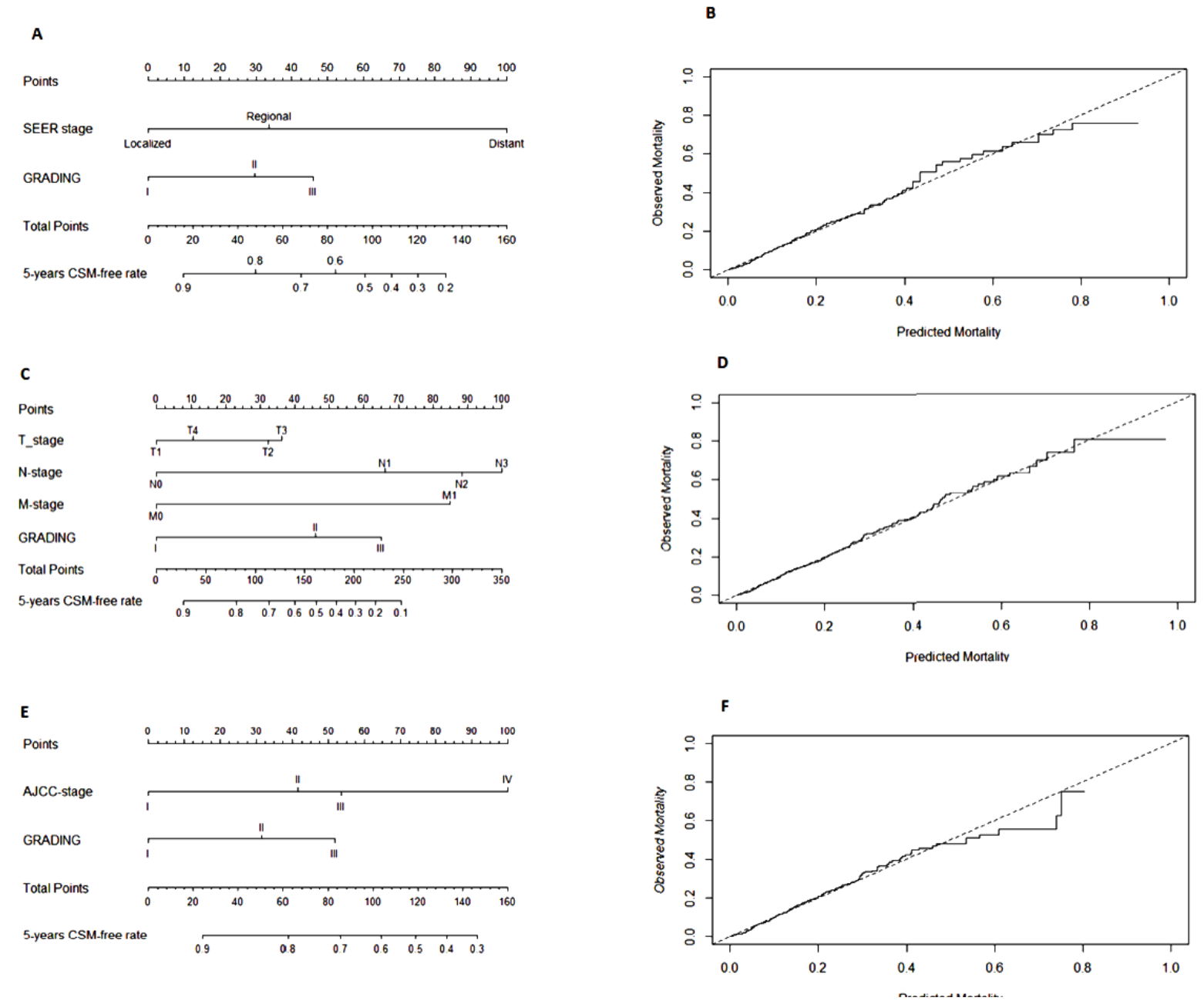

Figure 4: Nomograms predicting CSM-free rate 5 years after primary tumor excision using SEER stage (A), TNM classification (C) and AJCC stage (E) combined with TG. Calibration between predicted ( $x$ axis) and observed (y axis) 5-year CSM free rate for SEER stage (B), TNM classification (D) and AJCC stage (F) models.

The models with or without TG to predict 5-year CSM free mortality for the penile cancer were calibrated using nomograms (Figure 4, A-F). In nomograms based on SEER stage and TG, distant metastasis was the most powerful CSM predictor. Similarly, in nomogram based on AJCC stage and TG, AJCC stage IV PPSCC was the most powerful predictor respectively. However, in each nomogram, high TG was a less influential variable than SEER stage distant and AJCC stage IV. Nodal status N3 also had a powerful effect. However, Nodal status (N1 and N2) and TG II and III had an intermediate effect. $\mathrm{T}$ substage was an even less influential variable. The internal calibration done with nomogram shows a good relationship between predicted and observed rate.

\section{Discussion}

This study included 1304 cases from SEER registry that addresses $34.6 \%$ of the USA population and it's one of the largest cancer databases. ${ }^{9}$ Here, all penile cancer patients who underwent penile tumor excision were included and analysis was done taking their SEER stage, AJCC stage, TNM stage and combining tumor grade with them. We made an attempt to develop a simple prognostic rule that could be applied to the US men to predict the CSM after penile cancer. This study is done to know if findings in previous studies were consistent even after many years now. ${ }^{3-5}$

Penile cancer, though more common among elderly men (above 60 years), in small percentage it can also occur 
in younger males. ${ }^{10-12}$ In this study, we found it be more common in men above 60 years. Men who are unmarried, divorced or separated are more prone to the disease. Moreover, multiple sexual partners or history of sexually transmitted diseases or no use of barrier contraceptives are at risk. ${ }^{13,14}$ The disease may occur at any site, however, the glans penis is the most common primary site and well to moderately differentiated squamous cell carcinoma is the most common histological grade. ${ }^{11,15}$

Table 1: Clinical- pathological profile of patients with PPSCC

\begin{tabular}{|c|c|c|c|}
\hline \multicolumn{2}{|r|}{ Patients Characteristic } & \multirow{2}{*}{$\begin{array}{c}\text { Frequency } \\
64.66 \pm 14.38\end{array}$} & \multirow{2}{*}{ Percentage (\%) } \\
\hline Age & Mean \pm SD & & \\
\hline \multirow{9}{*}{ Age Group } & $20-29$ years & 7 & .5 \\
\hline & $30-39$ years & 58 & 4.4 \\
\hline & 40-49 years & 136 & 10.4 \\
\hline & $50-59$ years & 252 & 19.3 \\
\hline & 60-69 years & 347 & 26.6 \\
\hline & 70-79 years & 292 & 22.4 \\
\hline & $80-89$ years & 179 & 13.7 \\
\hline & 90-99 years & 32 & 2.5 \\
\hline & 100-109 years & 1 & .1 \\
\hline \multirow{4}{*}{ Race } & White & 1094 & 83.9 \\
\hline & Black & 126 & 9.7 \\
\hline & Other & 72 & 5.5 \\
\hline & Unknown & 12 & .9 \\
\hline \multirow{6}{*}{ Marital Status } & Single (never married) & 216 & 16.6 \\
\hline & Married (including common law) & 762 & 58.4 \\
\hline & Separated & 13 & 1.0 \\
\hline & Divorced & 116 & 8.9 \\
\hline & Widowed & 115 & 8.8 \\
\hline & Unknown & 82 & 6.3 \\
\hline \multirow{5}{*}{ Primary Site } & C60.0-Prepuce & 155 & 11.9 \\
\hline & C60.1-Glans penis & 483 & 37.0 \\
\hline & C60.2-Body of penis & 63 & 4.8 \\
\hline & C60.8-Overlapping lesion of penis & 55 & 4.2 \\
\hline & C60.9-Penis, NOS & 548 & 42.0 \\
\hline \multirow{3}{*}{ Grade } & Well Differentiated & 435 & 33.4 \\
\hline & Moderately Differentiated & 599 & 45.9 \\
\hline & Poorly Differentiated/ Undifferentiated & 270 & 20.7 \\
\hline \multirow{3}{*}{ SEER Stage } & Localized & 777 & 59.6 \\
\hline & Regional & 456 & 35.0 \\
\hline & Distant & 71 & 5.4 \\
\hline \multirow{4}{*}{ AJCC Stage } & Stage I/0 & 670 & 51.4 \\
\hline & Stage II & 298 & 22.9 \\
\hline & Stage III & 232 & 17.8 \\
\hline & Stage IV & 104 & 8.0 \\
\hline \multirow{4}{*}{ T Stage } & $\mathrm{T} 1 / \mathrm{Ta}$ & 740 & 56.7 \\
\hline & T2 & 328 & 25.2 \\
\hline & T3 & 214 & 16.4 \\
\hline & T4 & 22 & 1.7 \\
\hline \multirow{4}{*}{ N Stage } & $\mathrm{N} 0 / \mathrm{NX}$ & 1066 & 81.74 \\
\hline & N1 & 88 & 6.7 \\
\hline & $\mathrm{N} 2$ & 88 & 6.7 \\
\hline & N3 & 62 & 4.8 \\
\hline \multirow{2}{*}{ M Stage } & M0 & 1269 & 97.3 \\
\hline & M1 & 35 & 2.7 \\
\hline \multirow{4}{*}{ Type of surgery } & Local tumor excision & 372 & 28.5 \\
\hline & Simple/partial removal of primary site & 719 & 55.1 \\
\hline & Total removal of the primary site & 164 & 12.6 \\
\hline & Radical surgery & 49 & 3.8 \\
\hline
\end{tabular}


Table 2: Univariate and multivariate cox regression models to predict CSM in 1304 patients.

\begin{tabular}{|c|c|c|c|c|c|c|}
\hline \multirow{2}{*}{ Predictors(stage) } & \multicolumn{3}{|c|}{ Staging system alone } & \multicolumn{3}{|c|}{ Staging system + Grade } \\
\hline & HR (95\%CI) & p-value & $\%$ AUC & HR (95\%CI) & p-value & \% AUC \\
\hline SEER & & & 68.8 & & & 72.8 \\
\hline Regional vs. localized & $2.65(2.0-3.51)$ & $<0.001$ & & $2.18(1.63-2.91)$ & $<0.001$ & \\
\hline Distant vs. localized & 13.08(9.09-18.8) & $<0.001$ & & $10.17(7.0-14.74)$ & $<0.001$ & \\
\hline AJCC & & & 70.3 & & & 73.1 \\
\hline II vs. I & $2.60(1.83-3.69)$ & $<0.001$ & & $2.29(1.61-3.26)$ & $<0.001$ & \\
\hline III vs. I & $3.64(2.57-5.19)$ & $<0.001$ & & $2.91(2.04-4.15)$ & $<0.001$ & \\
\hline IV vs. I & $9.44(6.52-13.68)$ & $<0.001$ & & $7.35(5.02-10.75)$ & $<0.001$ & \\
\hline $\mathrm{T}:$ & & & 62.6 & & & 68.4 \\
\hline T2 vs. T1 & $2.20(1.63-2.97)$ & $<0.001$ & & $1.92(1.42-2.59)$ & $<0.001$ & \\
\hline T3 vs. T1 & $2.98(2.17-4.08)$ & $<0.001$ & & $2.36(1.71-3.26)$ & $<0.001$ & \\
\hline T4 vs. T1 & $2.10(0.85-5.17)$ & 0.106 & & $1.72(0.70-4.24)$ & 0.239 & \\
\hline $\mathrm{N}:$ & & & 67.0 & & & 72.3 \\
\hline N1 vs. N0 & $3.92(2.70-5.69)$ & $<0.001$ & & $3.11(2.13-4.56)$ & $<0.001$ & \\
\hline N2 vs. N0 & $5.01(3.53-7.12)$ & $<0.001$ & & $3.98(2.78-5.72)$ & $<0.001$ & \\
\hline N3 vs. N0 & $8.06(5.54-11.73)$ & $<0.001$ & & $6.37(4.34-9.36)$ & $<0.001$ & \\
\hline M1 vs. M0 & 9.38(6.07-14.49) & $<0.001$ & 54.9 & 7.37(4.75-11.44) & $<0.001$ & 66.8 \\
\hline Stage & & & 64.0 & & & \\
\hline II vs. I & $2.59(1.81-3.72)$ & $<0.001$ & & & & \\
\hline III vs. I & $4.37(2.99-6.41)$ & $<0.001$ & & & & \\
\hline TNM combined & & & 72.3 & & & 75.0 \\
\hline \multicolumn{7}{|l|}{$\mathrm{T}:$} \\
\hline T2 vs. T1 & $1.64(1.21-2.24)$ & 0.002 & & $1.57(1.15-2.14)$ & 0.0042 & \\
\hline T3 vs. T1 & $1.80(1.29-2.52)$ & 0.0005 & & $1.66(1.19-2.32)$ & 0.0029 & \\
\hline T4 vs. T1 & $1.30(0.52-2.32)$ & 0.574 & & $1.16(0.46-2.89)$ & 0.750 & \\
\hline \multicolumn{7}{|l|}{$\mathrm{N}:$} \\
\hline N1 vs. N0 & $3.04(2.06-4.47)$ & $<0.001$ & & $2.52(1.70-3.73)$ & $<0.001$ & \\
\hline N2 vs. N0 & $4.00(2.77-5.77)$ & $<0.001$ & & $3.43(2.38-4.97)$ & $<0.001$ & \\
\hline N3 vs. N0 & $4.92(3.21-7.55)$ & $<0.001$ & & $4.04(2.61-6.25)$ & $<0.001$ & \\
\hline M1 vs. M0 & $3.17(1.96-5.13)$ & $<0.001$ & & $3.27(2.01-5.31)$ & $<0.001$ & \\
\hline
\end{tabular}

Patients usually presented early when the disease is localized to the organ itself or at an early stage (AJCC stage I/O). Many efforts have been made to find the best and simple way to predict the CSM free rates and check the accuracy of predictors as to know the chances of survival, recurrence and the extent of the disease before the event happens.

In 2006, Kattan et al (2006) studied 175 Italian patients from 1998-2002 included detailed pathological variables (tumor thickness, growth pattern, grading, lymphatic/ tumor venous emboli, corpora cavernous/corpora spongiosum infiltration, urethral infiltration, regional LN treatment and pathological required LN involvement) to predict the 5-year CSM free rate for penile cancer. This study had $72.8 \%$ accuracy on the prediction of CSM rate. ${ }^{3}$ However, to find all these details were impractical, as most of the time all the details are not mentioned in the pathology report and it wasn't found to be useful in all setting.

Similarly, in 2011, Zini et al studied 856 American patients from 1988-2004 from 9 SEER cancer registries and claimed that the 5-year CSM free rates could be calculated in a simple way by just including SEER stage and grading in together. They found the SEER stage when combined with TG was $73.8 \%$ accurate in predicating CSM free mortality rate. ${ }^{5}$

In the present study, we compared the SEER stage, AJCC stage, and TNM stage with or without TG to predict the CSM free survival after PTE. Our model showed 


\section{BPKMCH}

the TNM stage had the highest accuracy $(72.3 \%)$ and when combined with TG the accuracy increased to $75 \%$. Similarly, the AJCC stage had an accuracy of $70.3 \%$ and when combined with TG, accuracy was $73.1 \%$. Similarly, SEER stage with TG had the least accurate predictability of CSM free rate compared to TNM stage with TG (72.8\% vs $75 \%)$. Relative to nomogram of Kattan et al and Zini et al our model is found to be more accurate to predict the CSM free rates because these are based on the SEER data. ${ }^{3,5}$

Our results are comparable to the results of the study conducted by Thuret et al. Analyzing their findings, TNM stage (78\%) was more accurate than AJCC stage (77.2\%) in predicting 5-year CSM free rates. When TG was

\section{NEPALESE JOURNAL OF CANCER (NJC)}

combined to both of the models, it definitely improved the accuracy of both the models and there was an only slight difference of accuracy $0.2 \%$ TNM stage with TG (80.7\%) versus AJCC stage with TG $(80.9 \%){ }^{4}$

Thuret et al included 1324 patients from 15 SEER registries from the year 1988-2006 with histological subtype of PPSCC (8070-8076). Then it represented 26\% of the US population. However, in this study, we included 1304 men from 16 SEER registries from year 2004-2011and all histological subtype of PPSCC (8050-8089). Now, it represents the $34.6 \%$ percent of the US population. Moreover, our sample size is comparable to the sample size of Thuret et al (1324) and larger than the study conducted by Zini et al (856) and Kattan et al (175).

Table 3: Univariate and multivariate competing risk regression models to predict CSM

\begin{tabular}{|c|c|c|c|c|}
\hline \multirow{2}{*}{ Predictors(stage) } & Staging system alone & & Staging system + Grade & \multirow{2}{*}{ p-value } \\
\hline & HR (95\%CI) & p-value & HR (95\%CI) & \\
\hline \multicolumn{5}{|l|}{ SEER } \\
\hline Regional vs. localized & $2.51(1.97-3.20)$ & $<0.001$ & $2.08(1.56-2.79)$ & $<0.001$ \\
\hline Distant vs. localized & $10.8(7.46-15.63)$ & $<0.001$ & $8.50(5.70-12.70)$ & $<0.001$ \\
\hline \multicolumn{5}{|l|}{ AJCC } \\
\hline II vs. I & $2.15(1.59-2.91)$ & $<0.001$ & $2.21(1.56-3.13)$ & $<0.001$ \\
\hline III vs. I & $3.38(2.51-4.56)$ & $<0.001$ & $2.79(1.94-4.00)$ & $<0.001$ \\
\hline IV vs. I & $7.48(5.30-10.55)$ & $<0.001$ & $6.15(4.13-9.15)$ & $<0.001$ \\
\hline \multicolumn{5}{|l|}{$\mathrm{T}:$} \\
\hline T2 vs. T1 & $2.10(1.57-2.83)$ & $<0.001$ & $1.62(1.24-2.12)$ & $<0.001$ \\
\hline T3 vs. T1 & $2.78(2.03-3.82)$ & $<0.001$ & $2.18(1.63-2.91)$ & $<0.001$ \\
\hline T4 vs. T1 & $1.95(0.78-4.90)$ & 0.160 & $1.96(0.98-3.92)$ & 0.056 \\
\hline \multicolumn{5}{|l|}{$\mathrm{N}:$} \\
\hline N1 vs. N0 & $3.73(2.59-5.37)$ & $<0.001$ & $3.08(2.10-4.53)$ & $<0.001$ \\
\hline N2 vs. N0 & $4.72(3.33-6.69)$ & $<0.001$ & $3.84(2.66-5.54)$ & $<0.001$ \\
\hline N3 vs. N0 & $6.75(4.57-9.98)$ & $<0.001$ & $5.44(3.63-8.15)$ & $<0.001$ \\
\hline M1 vs. M0 & $7.73(4.32-11.8)$ & $<0.001$ & $5.38(3.33-9.34)$ & $<0.001$ \\
\hline \multicolumn{5}{|l|}{ Stage } \\
\hline \multicolumn{5}{|l|}{ II vs. I } \\
\hline \multicolumn{5}{|l|}{ III vs. I } \\
\hline \multicolumn{5}{|l|}{ TNM combined } \\
\hline \multicolumn{5}{|l|}{$\mathrm{T}:$} \\
\hline T2 vs. T1 & $1.60(1.17-2.20)$ & 0.0034 & $1.52(1.11-2.08)$ & 0.009 \\
\hline T3 vs. T1 & $1.72(1.22-2.44)$ & 0.0022 & $1.56(1.10-2.21)$ & 0.013 \\
\hline T4 vs. T1 & $1.20(0.48-3.03)$ & 0.070 & $1.07(0.42-2.72)$ & 0.088 \\
\hline \multicolumn{5}{|c|}{ 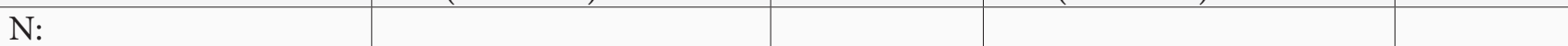 } \\
\hline N1 vs. N0 & $2.98(2.02-4.39)$ & $<0.001$ & $2.59(1.73-3.87)$ & $<0.001$ \\
\hline N2 vs. N0 & $3.84(2.62-5.62)$ & $<0.001$ & $3.37(2.29-4.94)$ & $<0.001$ \\
\hline N3 vs. N0 & $4.35(2.73-6.94)$ & $<0.001$ & $3.74(2.35-5.97)$ & $<0.001$ \\
\hline M1 vs. M0 & $2.96(1.66-5.29)$ & $<0.001$ & $2.93(1.65-5.19)$ & $<0.001$ \\
\hline
\end{tabular}


The current prognostic rule (TNM stage with TG), therefore, more accurately stratify the risk in patients with penile cancer than the use of other models. Such prognostic rule helps us to determine the consideration of adjuvant therapy and also the determination of the frequency of follow up.

As a number of penile cancer patients die of the noncancer-related cause, the Cox regression model may overestimate the true CSM free rates. To avoid such bias, we also used competing risk regression model. CSM free rate according to both models Cox regression and competing risk were calculated.

The CSM free rates according to Kaplan Meir based estimation was strikingly similar to cumulative incidence estimation by Fine and Gray methodology. Both curves superimposed each other. Thus, we relied on the Cox regression model to develop our prediction rule.

Our proposed four variables model TNM stage and TG though may appear more complex than two variable AJCC stage and TG, but this former model had better accuracy than later and this may provide the clinician an excellent ability to predict the CSM free rates.

Even though data collected in the SEER database is considered reliable it has some limitations. It may have variations in data entry or miscoding or incomplete data of various variables. The database didn't include information regarding co-morbidities like phimosis, smoking and other medical problems. Surgical details like margin status, which are important prognostic factors. Lack of central pathology review and accuracy of the available pathological report may inaccurately stage the disease. Likewise, Information regarding disease recurrence, adjuvant therapy, and long-term follow-up data is under addressed. ${ }^{16,17}$

The external validity of the nomograms is required for wide acceptance and clinical application of the observation. The ideal validation should be obtained in a prospective series of patients. This SEER database includes patients from USA and for external validation purpose, an additional patient from a second institution, or other countries Asia or South America with similar protocol with respect to surgery type indications need to be included. External validation was not done in this study.

\section{Conclusion}

TNM stage with TG and AJCC stage with TG appear to have comparable accuracy to predict the CSM free rate in patients with PPSCC, TNM stage with TG is the most accurate (75\%) method to predict the CSM free rates. Addition of TG variable definitely improved the accuracy of these prognostic model.

\section{References}

1. Correa AF, Handorf E, Joshi SS, Geynisman DM, Kutikov A, Chen DY, et al. Differences in Survival Associated with Performance of Lymph Node Dissection in Patients with Invasive Penile Cancer: Results from the National Cancer Database. J Urol. 2018;199(5):1238-44.

2. Althaf S, Narayanakar RP, Gangaiah DM, Dev K, Kurpad VP, Gurawalia J. Inguinal Lymph Nodes in Carcinoma Penis-Observation or Surgery? J Clin Diagn Res. 2016;10(1):XC01-XC4.

3. Kattan MW, Ficarra V, Artibani W, Cunico SC, Fandella A, Martignoni G, et al. Nomogram predictive of cancer specific survival in patients undergoing partial or total amputation for squamous cell carcinoma of the penis. J Urol. 2006;175(6):21038; discussion 8.

4. Thuret R, Sun M, Abdollah F, Budaus L, Lughezzani G, Liberman D, et al. Tumor grade improves the prognostic ability of American Joint Committee on Cancer stage in patients with penile carcinoma. J Urol. 2011;185(2):501-7.

5. Zini L, Cloutier V, Isbarn H, Perrotte P, Capitanio $\mathrm{U}$, Jeldres $\mathrm{C}$, et al. A simple and accurate model for prediction of cancer-specific mortality in patients treated with surgery for primary penile squamous cell carcinoma. Clin Cancer Res. 2009;15(3):1013-8.

6. Wen S, Ren W, Xue B, Fan Y, Jiang Y, Zeng C, et al. Prognostic factors in patients with penile cancer after surgical management. World J Urol. 2018;36(3):43540.

7. Qu XM, Siemens DR, Louie AV, Yip D, Mahmud A. Validation of predictors for lymph node status 
in penile cancer: Results from a population-based cohort. Can Urol Assoc J. 2018;12(4):119-25.

8. Fine JP, Gray RJ. A Proportional Hazards Model for the Subdistribution of a Competing Risk. Journal of the American Statistical Association. 1999;94(446):496-509.

9. Mena AC, Pulido EG, Guillen-Ponce C. Understanding the molecular-based mechanism of action of the tyrosine kinase inhibitor: sunitinib. Anticancer Drugs. 2010;21 Suppl 1:S3-11.

10. Thuret R, Sun M, Abdollah F, Budaus L, Shariat SF, Iborra F, et al. Competing-risks analysis in patients with T1 squamous cell carcinoma of the penis. BJU Int. 2013;111(4 Pt B):E174-9.

11. Nam JK, Lee DH, Park SW, Kam SC, Lee KS, Kim $\mathrm{TH}$, et al. Clinicopathologic Characteristics and Treatment Outcomes of Penile Cancer. World J Mens Health. 2017;35(1):28-33.

12. Sun M, Djajadiningrat RS, Alnajjar HM, Trinh QD, Graafland NM, Watkin N, et al. Development and external validation of a prognostic tool for prediction of cancer-specific mortality after complete locoregional pathological staging for squamous cell carcinoma of the penis. BJU Int. 2015;116(5):734-43.
13. Chalya PL, Rambau PF, Masalu N, Simbila S. Tenyear surgical experiences with penile cancer at a tertiary care hospital in northwestern Tanzania: a retrospective study of 236 patients. World J Surg Oncol. 2015;13:71.

14. Torbrand C, Wigertz A, Drevin L, Folkvaljon Y, Lambe M, Hakansson U, et al. Socioeconomic factors and penile cancer risk and mortality; a populationbased study. BJU Int. 2017;119(2):254-60.

15. Jayaratna IS, Mitra AP, Schwartz RL, Dorff TB, Schuckman AK. Clinicopathologic characteristics and outcomes of penile cancer treated at tertiary care centers in the Western United States. Clin Genitourin Cancer. 2014;12(2):138-42.

16. Duggan MA, Anderson WF, Altekruse S, Penberthy L, Sherman ME. The Surveillance, Epidemiology, and End Results (SEER) Program and Pathology: Toward Strengthening the Critical Relationship. Am J Surg Pathol. 2016;40(12):e94-e102.

17. Yu JB, Gross CP, Wilson LD, Smith BD. NCI SEER public-use data: applications and limitations in oncology research. Oncology (Williston Park). 2009;23(3):288-95. 